\title{
Surgical Chest Conditions
}

\author{
By Dr. J. C. VAN DER SPUY, M.B., B.Ch., Dip. in Surg. \\ Thoracic Surgeon, Pretoria General Hospital. \\ Lecture given at the Northern Transval Branch Post-Graduate Course on Chests, on Saturday, $28 t h$ May.
}

Mr. President, Madam Principal, Ladies and Gentlemen, I wish to thank you for having asked me to address you on this very important occasion.

I have been requested to talk on the subject of Cardiac Surgery. This I do very gladly because this is probably the branch of surgery which demands the closest co-operation between the various members of the team including the physiotherapist; and as the most common and probably one of the most serious complications to follow open-heart surgery is related to the broncho-pulmonary system, it is absolutely essential that the physiotherapist be fully aware of his or her most important responsibilities as a member of a team working together in the closest harmony with only one single goal in mind. It is not my intention to discuss here with you today the physiotherapeutic aspects of cardiac surgery, but rather to give you a broad view on cardiac surgery as practised today.

\section{Heart lesions requiring surgical treatment}

The various heart lesions which require surgical treatment include both congenital and acquired defects and include stenosis of the mitral, aortic, pulmonary and tricuspid valves, regurgitation of the mitral and aortic valves, defects in the interventricular and interarterial septa, various combinations of valvular stenosis with septal defects, lesions of the main blood vessels in close proximity to the hearte.g. patent ductus arteriosus and coarctation of the aortaand lesions in the coronary arterial supply to the myocardium. Many of these lesions can be more or less completely cured with present day surgical techniques, but the correction of other-e.g. mitral and aortic regurgitation and lesions of the coronary arteries-are still in the experimental stage.

\section{Thoracic incisions}

Various thoracic incisions are employed for approaching the heart. The most common and firmly established incisions enter the right or left chest cavity by means of an incision along the line of a rib and through an intercostal space or through the bed of the corresponding rib which can either be removed or left intact. Such incisions may be postero-lateral or antero-lateral and depending on their situation will extend through the latissimus dorsi, serratus anterior and pectoralis muscles. The anterior trans-sternal incision may be unilateral or bilateral and extends transversely through the sternum and the corresponding intercostal space on one or both sides. The more recently introduced, and now more commonly used, vertical trans-sternal incision bisects the sternum from the suprasternal notch to the xyphoid process. The main advantages of this incision are that it can be done more quickly and is less painful postoperatively, with the result that the patient can co-operate more readily with the physiotherapist.

\section{Cardiac approaches}

The lesion may be reached by means of various cardiac approaches. The mitral valve, for example, is reached via the left atrium, whereas the tricuspid valve and an interatrial septal defects are approached via the right atrium and a ventricular septal defect is reached through an incision in the right ventricle. The approach to the aortic and pulmonary valves is either through the corresponding ventricle or vessel. The size of the cardiac incision may be such as to accommodate only a finger or a special instrument in cases of closed heart operations, or the incision may be sufficiently large so as to allow of the surgery being carried out under direct vision.

\section{Two main techniques}

Two main techniques are used in the performance of heart operations depending on whether the operation is of the closed-heart type or of the open-heart type.

\section{Closed heart operations}

In closed heart operations the valve, such as the mitral and tricuspid valves, is reached via an incision in the left and in the right atrium respectively of sufficient size to accommodate only an index finger, which is used to force open the valve along its fused commissures. Similarly, a valve dilator can be passed through a small wound in the right or in the left ventricle into the pulmonary or the mitral and aortic valves, which are forced open blindly. This closed transventricular approach is today the operation of choice in cases of mitral stenosis and under certain circumstances also in aortic stenosis; in the former case the tip of the instrument in the mitral valve is controlled by means of a second finger which is introduced into the left atrium through a small wound in the left auricular appendage.

\section{Open heart operation.}

Since the first successful intra-cardiac operation, twelve years ago, for mitral stenosis, much experimental work, chiefly in the U.S.A., has made it possible to explore the interior of the heart under direct vision employing in the first instance hypothermia and followed a few years later by the use of the heart-lung machine. Hypothermia is in use today in many cardiac centres for the repair chiefly of atrial septal defects and for pulmonary valvotomy via the pulmonary artery. At the normal body temperature of $37^{\circ} \mathrm{C}$ the brain can stand, without damage, anoxia for a period of three to four minutes. When, however, the body temperature has been reduced, by placing the patient for example in a tub with ice water, to a temperature of $29^{\circ} \mathrm{C}$ the brain will withstand a period of anoxia of about eight to ten minutes. Under the latter circumstances the superior and inferior vena cava can be clamped off, the inflow into the right atrium thus eliminated and the right atrium be opened and a septal defect sutured under direct vision, provided that the operation of suturing the defect be completed within eight to ten minutes. Under the same set of circumstances the pulmonary artery immediately distal to the pulmonary valve can be incised and the commissures of the stenosed pulmonary valve be incised under direct vision.

\section{Heart-lung machine.}

The main drawback of open-heart surgery under hypothermia is the very limited period of time at the surgeon's disposal to repair more complicated intra-cardiac defects. 1 For such defects the heart-lung machine is used. The principle of the machine is the following.

Venous blood is sucked out of the superior and inferior vena cava by means of cannulae which are passed into these veins via the right atrium. By means of tapes placed around the superior and inferior vena cava these vessels are constricted around the catheters with the result that no venous blood can reach the right atrium. The venous blood is pumped by means of a mechanical pump to a mechanical lung or oxygenator, from where it is pumped by a second pump to the femoral artery. This oxygenated or arterialised blood reaching the femoral artery passes retrograde along the aorta against the closed aortic valves, and from the aorta along all its branches. This blood passes through the whole body and returns via the superior and inferior vena cava whence it is again drained to pump No. 1 and the oxygenator. The heart is therefore empty except for coronary arterial blood which drains via the coronary sinus into the right atrium.

With the aid of the heart-lung machine, operations on the open heart can be done for periods of as long as two hours. The heart-lung machine is most commonly used for ventricular septal defects, but is also commonly employed for (Continued on page 5) 


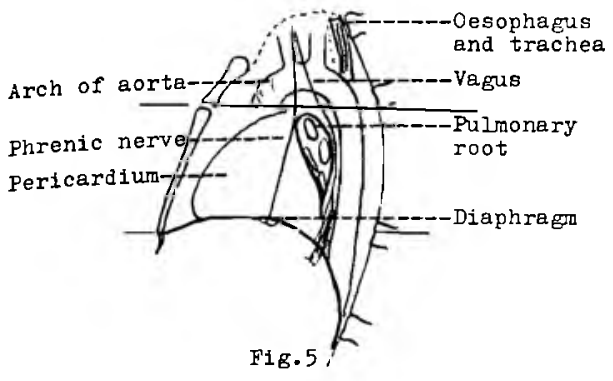

Structures seen through the left mediastinum.

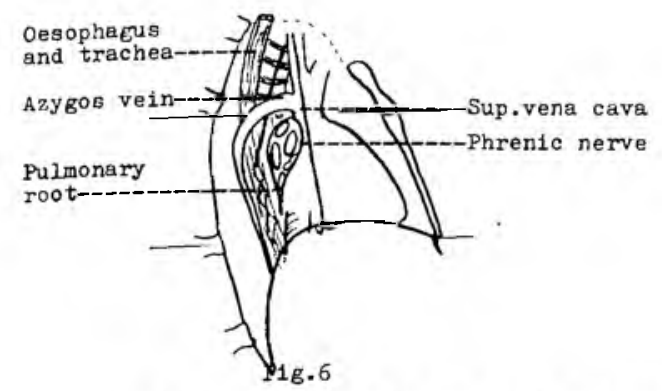

Structures seen through the right mediastinum.

The Trachea and Lungs

The trachea begins in the neck at the lower end of the larynx. It enters the thorax opposite the upper border of the manubrium sterni and terminates at the lower border of the manubrium opposite the 4th thoracic vertebra by dividing into a right and left bronchus. Its thoracic part therefore lies in the superior mediastinum.

The trachea, being an elastic structure, elongates during inspiration and its bifurcation may thus descend to the level of the body of the fifth or even the sixth thoracic vertebra.

\section{Bronchi}

Each bronchus passes downwards and sidewards to the hilum of the corresponding lung and thence downwards in the substance of the lung towards its base. The primary bronchi can therefore be divided into extra pulmonary and intra pulmonary portions, kept patent by rings-and plates of cartilage, respectively.

The right bronchus is shorter than the left bronchus, it is more vertical than the left and is slightly wider. It gives off one branch, which arises close to the hilum of the lung and is called the eparterial bronchus because it originates immediately above the point where the right pulmonary artery crosses in front of the bronchus.

The left bronchus gives off no branches in the hilum.

\section{The Lungs}

The lungs are a pair of comparatively light organs. The elasticity of the healthy lung substance is remarkable.

The lungs, when healthy, lie free in the cavity of the chest and are attached only by their roots.

Each lung presents for examination an apex, a base, a costal surface and a medial surface separated by anterior, posterior and inferior borders.

The apex rises into the root of the neck for an inch and a half above the level of the anterior part of the first rib.

The base of each lung has a semi lunar outline and is adapted to the upper surface of the diaphragm.

\section{Lobes of the Lungs}

The left lung is divided into two lobes by a long deep fissure which penetrates its substance to within a short distance of the hilum.

An oblique fissure and a horizontal fissure divides the right lung into three lobes.

Just as the lobes constitute major segments of the lung with their own bronchial, arterial and venous supply, so do the bronchi within a lobe branch and rebranch to supply smaller and smaller segments of lung tissue. In practice only the major broncho pulmonary segments supplied by tertiary bronchi are named or numbered.

\section{(Continued from page 6)}

atrial septal defects, aortic stenosis and regurgitation, mitral regurgitation and other complicated congenital heart lesions. Owing to the marked drawback of having to work on an actively beating but empty heart, the technique of injecting a $2 \frac{1}{2} \%$ solution of potassium citrate in arterialised blood into the ascending aorta of a patient on the heart-lung machine was introduced by Melrose about five years ago, as this procedure brings about cardiac standstill which markedly facilitates the performance of the intra-cardiac operation. More recently it has been shown that be merely clamping off the ascending aorta and so depriving the heart of oxygen spontaneous cardiac standstill will be produced which will recover again spontaneously after removal of the clamp.

We shall now show you a film, very kindly lent to us by Winthrop Products, of an operation performed by $\mathrm{Dr}$ Jerome Kay in the U.S.A., employing the principle described above of anoxic cardiac arrest with incision of the left ventricle to remove a tumour from the cavity of this ventricle, extending into both the left atrium and aorta via the mitral and aortic valves.

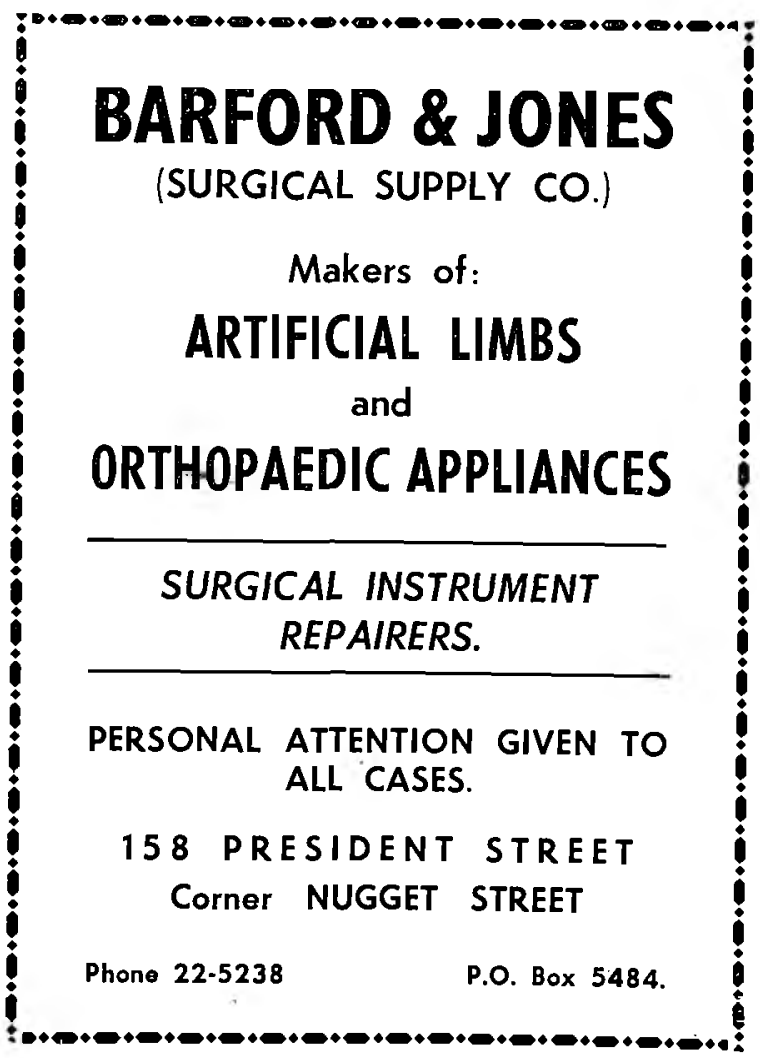

